\title{
Recherche en santé et données personnelles : la nouvelle donne
}

> Le point de vue développé ici est celui d'une juriste spécialiste des systèmes d'information de santé et de la protection des données. Après dix ans passés à la CNIL, j'ai rejoint I'Inserm en 2014 et suis en charge du pilotage de la politique de protection des données dans l'établissement.

L'essor des sciences et les technologies numériques a fait exploser la quantité et l'hétérogénéité des informations disponibles. Aux données scientifiques issues de la recherche clinique et en santé s'ajoutent des données médico-administratives recueillies initialement à des fins gestionnaires et des données issues de l'utilisation d'internet, de smartphones ou d'objets et de dispositifs médicaux connectés.

Ces données représentent un potentiel de contribution à la santé individuelle et collective dont la concrétisation dépendra de la validation par la recherche des méthodes et des outils et de la capacité à organiser le développement d'un écosystème facilitant l'accès et l'exploitation des données dans le respect de la vie privée des personnes et avec la rigueur et l'éthique, indispensables à la confiance numérique. C'est pourquoi l'Inserm s'implique fortement dans l'organisation, la gestion et l'accès à ces données sensibles pour les communautés de recherche. Dans le prolongement du rôle d'opérateur d'extraction et de mise à disposition des données du Système national des données de santé (SNDS) pour des traitements mis en œuvre à des fins de recherche, d'étude ou d'évaluation, que l'Inserm s'est vu confier par le décret relatif au SNDS, l'effort actuel de l'I'Inserm porte sur la mise en place d'une infrastructure nouvelle afin d'optimiser l'utilisation de ces données à des fins de recherche. La mutualisation et la combinaison des forces et des compétences permettront d'être à un puissant levier d'innovation scientifique dans le respect de la réglementation et de la sécurité des données.

Rappelons à cet égard que si le cadre juridique applicable à l'utilisation des données de santé à des fins de recherche n'est pas nouveau et présente déjà une dimension européenne, il est actuellement en cours de refonte tant au plan européen que national et présente un caractère complexe.

Le Règlement européen 2016/679 relatif à la protection des personnes physiques à l'égard du traitement des données à caractère personnel et à la libre circulation de ces données (RGPD) du 27 avril 2016, qui sera applicable le 25 mai 2018, marque un changement de paradigme. Alors que le régime de protection des données actuel repose en grande partie sur l'existence de formalités administratives préalables, le RGPD reposera sur une logique de conformité et de responsabilité des acteurs qui devront être en mesure de démontrer de façon continue la

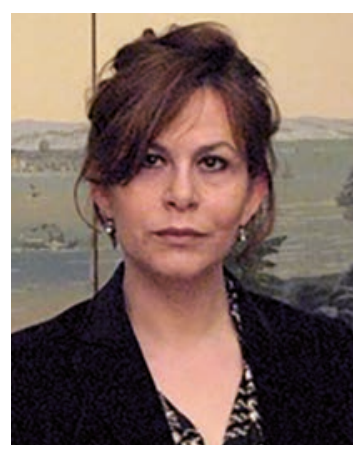

Responsable de la conformité en matière de protection des données, Inserm, 101 , rue de Tolbiac, 75654 Paris Cedex 13, France. frederique.lesaulnier@ inserm.fr

conformité des traitements qu'ils mettent en œuvre à la réglementation. Ils seront soumis à des obligations nouvelles lourdement sanctionnées et devront mettre en place une véritable politique de gouvernance des données à caractère personnel (désigner un délégué à la protection des données, tenir un registre de tous les traitements, mener des études d'impact pour les traitements qui présentent des risques élevés pour la vie privée, documenter tous les processus en interne qui permettent de démontrer la conformité, notifier les failles de sécurité...).

En outre, il faudra articuler le RGPD avec le droit national dans la mesure où il laisse d'importantes marges de manœuvre aux États membres qui peuvent maintenir ou adopter des spécificités nationales pour certains types de traitements parmi lesquels figurent les traitements qui portent sur les données de santé, les données génétiques, le numéro d'identification national et les traitements à des fins de recherche scientifique. C'est la raison pour laquelle un projet de loi d'adaptation de la loi du 6 janvier 1978 au RGPD est en cours de discussion au Parlement, qui devra être articulé avec le cadre national applicable à la recherche en santé issu notamment de la loi du 26 janvier 2016 de modernisation de notre système de santé qui crée le SNDS, ainsi que l'ensemble des dispositions du code de la santé publique applicables.

Dans ce contexte, la sécurité est une exigence dotée d'une force accrue et c'est particulièrement vrai dans le cas de maladies rares où le risque de ré-identification des personnes est accru. II en va de même du rôle des patients ou des personnes à l'origine des données qui est majeur. Pourtant, l'exigence d'une information 
individuelle spécifique à chaque projet issu de ces données et/ou échantillons est difficilement compatible avec les projets reposant sur une réutilisation secondaire renouvelée et réitérée de données ou d'échantillons biologiques faiblement identifiants, collectés à cette fin. C'est pourquoi, il est indispensable de concevoir des modalités d'exercice des droits souples et dynamiques, susceptibles de permettre aux infrastructures de répondre aux enjeux d'une meilleure compréhension des mécanismes pathologiques, tout en garantissant une meilleure maîtrise par les personnes des données et des échantillons biologiques qui les concernent. À cet égard, il faut saluer l'évolution de la doctrine de la CNIL sur ce point, illustrée par le projet de méthodologie de référence MR004 soumis à la concertation des acteurs représentatifs de la recherche. Celle-ci admet que l'information puisse être considérée comme valablement délivrée dès lors que les personnes avaient été informées de la réutilisation possible de leurs données et/ou échantillons biologiques lors de la collecte initiale et que l'information initiale renvoie à un dispositif spécifique d'information auquel les personnes pourront se reporter avant la mise en œuvre d'un nouveau traitement (par exemple un site internet). Ce type d'approche apparaît de nature à favoriser l'utilisation des données en recherche tout en préservant l'autonomie des patients qui deviennent de véritables parties prenantes au projet. Il est également souhaitable que les patients ou leurs représentants intègrent les structures de gouvernance des infrastructures et participent à l'élaboration du projet, des principes directeurs et des procédures d'accès mises en place. $\diamond$

Research in health and personal data: the new situation LIENS D'INTÉRÊT

L'auteur déclare n'avoir aucun lien d'intérêt concernant les données publiées dans cet article.

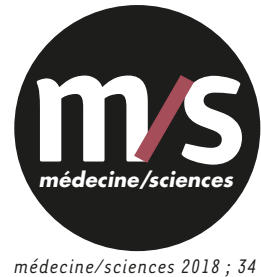
(hors série $n^{\circ} 1$ ) : $28-29$

\section{L'implication du malade expert dans toutes les étapes du développement des thérapies : état des fieux}

Karen Aïach
> Je suis la mère d'une petite fille, atteinte d'une maladie de surcharge lysosomale, le syndrome de Sanfilippo de type A, maladie neurologique et mortelle au début de l'adolescence du patient. J'ai aussi goûté à la vie associative au sein d'Eurordis et au sein d'une association que j'ai créée autour de la maladie de Sanfilippo. J'ai eu une expérience réglementaire au sein du Comité pédiatrique de l'Agence européenne du médicament et d'un comité de protection des personnes. J'ai décidé il y a quelques années de créer une société de biotechnologie pour porter le projet thérapeutique que j'avais mis en œuvre avec d'autres et essayer de mettre au point un traitement pour la maladie de Sanfilippo.

Les réglementations essentielles et fondatrices qui nous permettent d'être ici aujourd'hui ont été en grande partie initiées par des patients, leurs familles et ceux qui les entourent, comme les chercheurs et les médecins. Ces acteurs ont agi dans bien des champs différents, à commencer par la sensibilisation à la maladie rare et par l'éducation. La loi Hôpital, patient, santé et territoire de 2009 fait d'ailleurs essentiellement référence à cette dimension éducative. Le

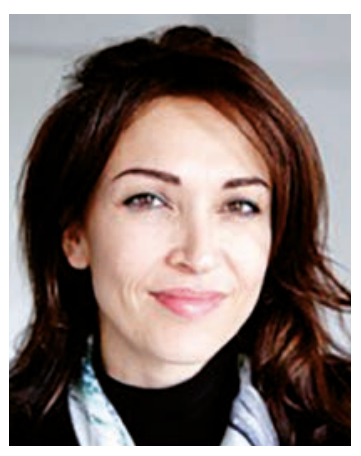

Fondatrice et Directrice Générale, Lysogene, 18 , rue Jacques Dulud, 92200 Neuilly-sur-Seine, France.

karen.aiach@lysogene.fr

patient se retrouve au cœur de l'innovation thérapeutique, non seulement comme objet d'étude, mais aussi comme acteur de l'éducation thérapeutique.

Un vaste écosystème s'est développé autour des patients et des familles, vivace et de grande qualité. Diplômée de I'ESSEC, j'ai commencé ma carrière dans une société américaine et ait travaillé dans le consulting. En arrivant dans l'univers des maladies rares, j'ai été frappée par la qualité des profils et des processus collaboratifs mis en œuvre par les différents acteurs. 